\title{
An Electric Field-Based Approach for Quantifying Volumes and Radii of Chemically Affected Space
}

\author{
Austin M. Mroz, Joshua Davis, Christopher H. Hendon* \\ Department of Chemistry and Biochemistry, University of Oregon, Eugene, OR, 97403 \\ * chendon@uoregon.edu \\ Density Functional Theory, Volume, Surface Area, Atomic Radius, Molecular volume
}

\begin{abstract}
Chemical shape and size play a critical role in chemistry. The van der Waal (vdW) radii, a familiar manifold used to quantify size by assuming overlapping spheres, provides rapid estimates of size in atoms, molecules, and materials. However, the vdW method may be too rigid to describe highly polarized systems and chemical systems that stray from spherical atomistic environments. To deal with these exotic chemistries, numerous alternate methods based on electron density have been presented. While each boasts inherent generality, all define the size of a chemical system, in one way or another, by its electron density. Herein, we revisit the timeless problem of assessing sizes of atoms and molecules, instead through examination of the electric field produced by them. While conceptually different than nuclei-centered methods like that of van der Waal, the field assesses chemically affected volumes. This approach implicitly accounts for long-range fields in highly polar systems, predicts that cations should affect more space than neutral counterparts.
\end{abstract}

\section{INTRODUCTION}

Chemical shape and size, and accompanying surface area and volume, are fundamental properties that govern a wealth of intermolecular phenomena in atoms, molecules, and materials ${ }^{1-7}$. Determination of chemical volumes and surfaces area are, however, obscured by the definition of the atomic "surface".8,9 Today, there are numerous approaches to quantify chemical size and, while the van der Waals method is certainly the most prevalent, alternatives have been developed through a synergy of experiment and theory. ${ }^{9}$ Understanding the limitations and applicability of these alternatives is important because numerous advanced measurements implicitly rely on size in some form (e.g., specific surface area of gas molecules used in surface area and volumetric measurements of porous materials within the Brunauer-Emmett-Teller (BET) formalism ${ }^{10}$, quantifying void-space docking sites in enzymes $^{11,12}$, and so forth). Hence, there remains intrinsic value in revisiting this age-old problem: just how big are molecules?

Initial experimental measurements of atomic size were performed by Meyer in $1870,{ }^{13}$ where he identified a relationship between material density and atomic size, and obtained a periodic trend in atomic volumes. These values were later refined by Bragg ${ }^{14}$ and Pauling15, who developed methods for assessing atomic radii through X-ray scattering. In two separate works, Bondi and Batsanov revisited the radii presented by Pauling and Bragg, and it is these works that are synonymous with the "van der Waals (vdW) radii" of atoms and ions. ${ }^{16-18}$ An alternate approach was taken by Alvarez, ${ }^{19,20}$ and Biswas and Ghosh, ${ }^{21}$ who extracted atomic radii using statistical analyses of online databases. The generality of this approach is, however, limited because of the uncertainty for chemical environments not represented in the empirical data, ${ }^{9}$ for systems featuring regions of high polarity, ${ }^{22-24}$ molecules with elongated bonds, ${ }^{25-27}$ and other exotic chemistries.

First principal simulations have provided yet another alternative, boasting inherent generality to recover volumetric data of both known and undiscovered molecules from their computed electronic structures. ${ }^{28}$ Early examples of these calculations were presented by Slater who employed the maximum radial density of outermost single particle wavefunctions to define atomic radii, ${ }^{29-31}$ and several other related methods have also been reported. ${ }^{32-37}$. Later, Bader computed elemental size at the Hartree-Fock level of theory ${ }^{38}$. There, the surface of a chemical system was defined by an electron density cutoff of $0.002 e$ bohr $^{-3}$. This cutoff was refined by Boyd ${ }^{39}$ to be $0.001 e$ bohr $^{-3}$ within the DFT construct $^{40,41}$, with the justification that any smaller value of electron density would result in a negligible change in calculated radii. Finally, Rahm and Hoffmann furthered Boyd's work, applying the method to atomic ions using electron densities obtained from hybrid $\mathrm{DFT}^{9}$ (PBE0 $^{42-44}$, with a large basis set).

Yet the use of electron density alone poses problems for modeling cations, which certainly interact with their surroundings beyond their electron cloud; they create a large electric field. ${ }^{45}$ With this in mind, we thought to revisit the size quantification problem through examination of electrostatic potential and its derivative, the electric field. While other approaches concern the space that a chemical system occupies, an electric field description captures the volume that a chemical system affects. In this regard, both cations and anions should be larger than their charge neutral counterparts (i.e. they affect more space), polar bonds should produce larger 
fields than non-polar analogues, and size/shape should be affected by external fields. Thus, an electric field metric should provide a conceptually different description of chemical size, and a unique approach for defining the edge of a chemical system. Herein, we explore the generality and implications of quantifying chemical size through examination of atomic and polyatomic electric fields, and provide some examples of the utility of this approach.

\section{RESULTS AND DISCUSSION}

Method Description. To sample the electric field, we have developed a post-electronic structure processing software, STREUSEL (Structure Topology REcovery Using Sampling of the ELectric field) ${ }^{46}$, which computes chemically "affected" volumes and surface areas of atoms, molecules, and materials. Since the electric field is defined as the negative gradient of the electrostatic potential it is highly sensitive to subtle changes in polarization. Here, the edge of the chemical system is defined as the point in space where there is near-zero variance in the electric field magnitude. Conventional DFT and $a b$ initio calculations return reliable electrostatic potential values on the order of $10^{-6} \mathrm{eV}$ (i.e. $\mathrm{O}\left(10^{-6} \mathrm{eV}\right)$, or $2.3 \times 10^{-5} \mathrm{kcal} \mathrm{mol}^{-1}$ ), thus we consider a change of less than $10^{-5} \mathrm{eV}\left(2.3 \times 10^{-4} \mathrm{kcal}\right.$ $\mathrm{mol}^{-1}$ ) to be conservatively precise. Conceptually, as cutoff decreases more vacuum space becomes included in the regions associated with the molecule ${ }^{37,38}$, and while it is undoubtedly a variable, we justify our $10^{-5} \mathrm{eV}$ potential cutoff thermodynamically; the energy cutoff is on the order of $k_{\mathrm{B}}$, $\mathrm{O}\left(10^{-5} \mathrm{eV} \mathrm{K}^{-1}\right)$. For reference, a typical van der Waals interaction is on the order of $0.956-1.912 \mathrm{kcal} \mathrm{mol}^{-1,47,48}$ our cutoff accounts for fluctuations in the third decimal place, in principle providing sensitive, but still experimentally measurable information.

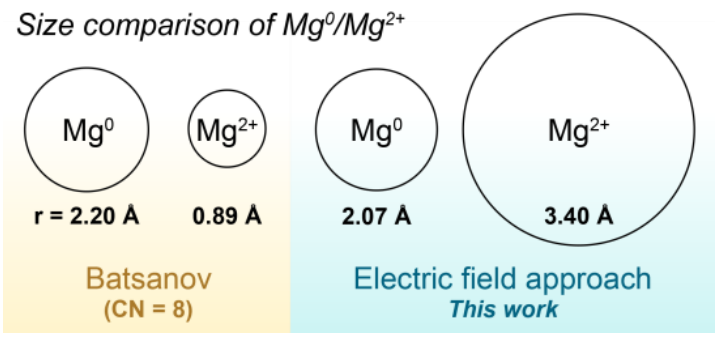

Figure 1 Comparison of atomic radii of $\mathrm{Mg}$ and $\mathrm{Mg}^{2+}$ between the Batsanov metal compressibility method (Coordination Number, $\mathrm{CN}=8$ innersphere ligands) and the electric field-based method presented in this work (radial line drawn from CCSD-full/aug-ccpVTZ data). See Figure S3 for a comparison of other size comparisons of $\mathrm{Mg}$ and $\mathrm{Mg}^{2+}$.

To employ this cutoff, one must first sample the electrostatic potential generated from a DFT or $a b$ initio calculation, with the density computed at a discrete number of volumetric pixels (voxels). Like the field cutoff, there is a dependence on the size of the voxels and computed size, coming at a trade-off between time-to-solution and voxel resolution, see Figure S1. From these data, a voxel of volume $0.008 \AA^{3}$ yields a desirable balance between computation time and volumetric resolution, while being sufficiently high fidelity to describe rapid changes in field across conventional chemical bonds, $O(\AA)$.
To illustrate the conceptual difference between our approach, we present the $\mathrm{Mg}^{0}$ and $\mathrm{Mg}^{2+} \mathrm{vdW}$ radii alongside the electric field radii computed using CCSD-full ${ }^{49} /$ aug-cc-pVTZ ${ }^{50}$, Figure 1. Conventional chemical tenets suggested by Batsanov would indicate that $\mathrm{Mg}^{2+}$, and cations in general, are smaller than their neutral counterparts (for elemental examples see Refs. 48-53, Table S1, and Figure S3 for a Mg-specific example). Yet, from an electrical field perspective, $\mathbf{M g}^{2+}$ is significantly larger than $\mathrm{Mg}^{0}$, because the electric field ultimately depends on the ratio of the number of protons and number of electrons. In other words, the area affected by a cation should be large, while the density of electrons is appreciably small. Hence, the $\mathrm{Mg}^{2+}$ example serves as an illustration that the field-defined size is not "atomic size" in the conventional sense and provides different insights than conventional atomic size models. The computed volume, however, should heavily depend on familiar quantum chemical variables such as functional, basis set, density grid size, etc. We will explore the other parameters throughout this paper.

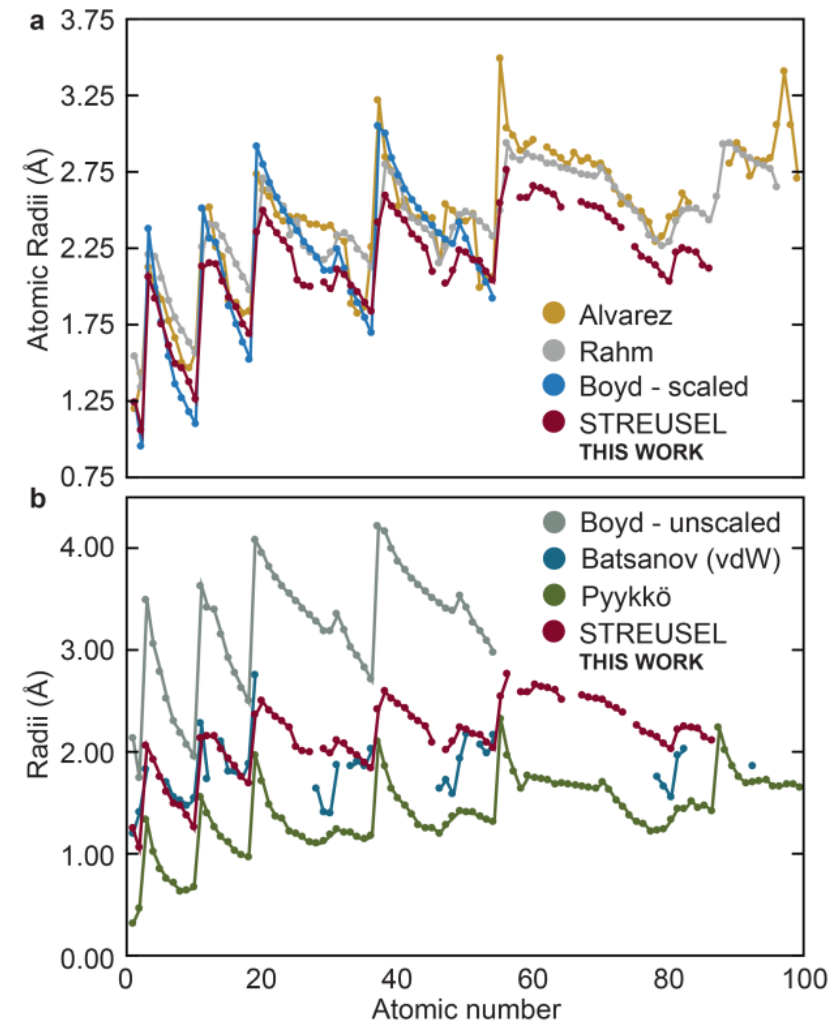

Figure 2 A comparison of atomic radii recovered using various conventional size metrics. a) Sizes computed from STREUSEL are similar to other electronic structure-based methods, and b) comparable to the vdW (Batsanov) sizes. Alternative size metrics (singly-bound data are presented for Pyykkö, and Boyd's unscaled approach) define the upper and lower limits.

Atoms and ions. Most sizing methods delineate between charged and charge neutral chemical systems, and free atoms and those in bonded environments. For example, the Pyykkö family of atomic radii ${ }^{57}$ only apply to covalently bonded systems, while the vdW radii presented by Bondi are reserved for charge neutral atoms. To contrast our method to these and other reported approaches we compute the periodic table of elements using CCSD-full ${ }^{58-61} / \mathrm{SDD}$-all as implemented in 
Gaussian0962 (see Figure S4 for a periodic table of elements), alongside some comparable and widely used methods, Figure 2. The basis was selected to both enable tractable calculations, with an ideal tradeoff between number of basis functions and accuracy, in addition to offering consistency between elements.

Generally, the atomic radius computed from the electric field is comparable with other electronic structure-based approaches (those presented by Alvarez, ${ }^{19,20}$ Boyd $^{39}$ and Rahm $^{9}$, Figure 2a). The largest divergence occurs at high atomic numbers (f-block elements), where the STREUSEL size predicts that a reduced volume and radius compared to electronic density methods. This could be due to difficulties in describing high quantum number electrons within DFT (i.e., they become diffuse, so the field change is small), but also could be limited by basis set.63 For the most part, however, our approach seems to predict similar size trends for free neutral atoms. Figure $\mathbf{2 b}$ reveals larger deviations between compared methods; Boyd's unscaled DFT-derived sizes, and Pyykkö radii for singly-bonded atoms bracket the radii of neutral elements. Pyykkö's singly-bound radii serves as an important reminder that the local environment plays a significant role in determining the interatomic radii. We would expect that the electric field produced by atoms in interacting environments would also deviate from the neutral sizes, Figure 2.
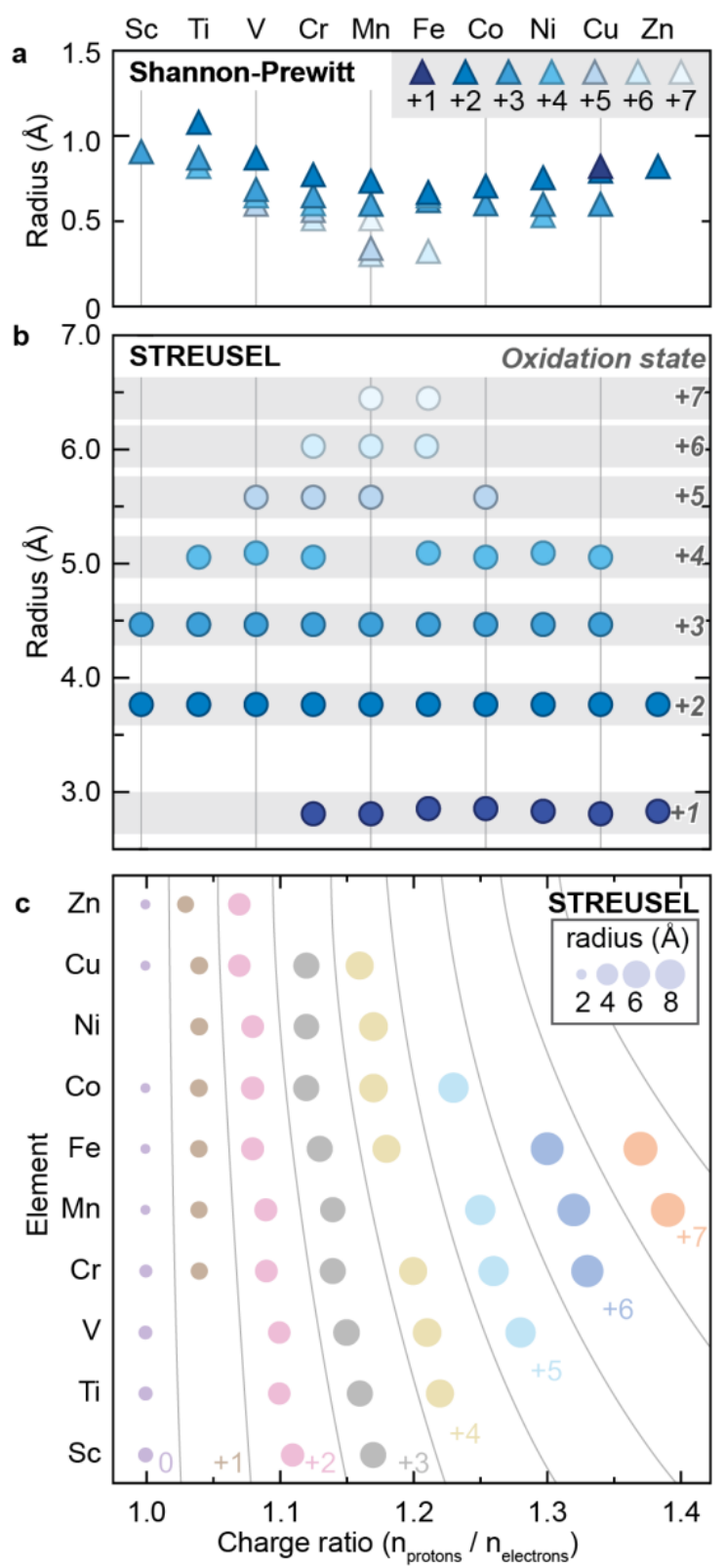

Figure 3 A comparison of ionic radii in the d-block. a) the Shannon-Prewitt sizes depend on oxidation state, and show a reduced size for mid-block elements (computed for 8 -inner sphere ligands). b) The electric field sizes are computed using the free ion and show a general relationship in radius to oxidation state, or $\mathbf{c}$ ) the core-charge to valence electron ratio. A complete periodic table comparison is presented in Figure S5.

The other striking conclusion is that the vdW radii seem to align well with electric field-derived sizing for the first 20 atoms, with subtle deviations in heavier main group elements. Indeed, the general shape of the predicted atomic sizes using STREUSEL seems to obey a trend common to electronic structure-based methods (per Figure 2a). It is rather the vdW radii that appear to predict reduced volumes for Groups 10 13. 

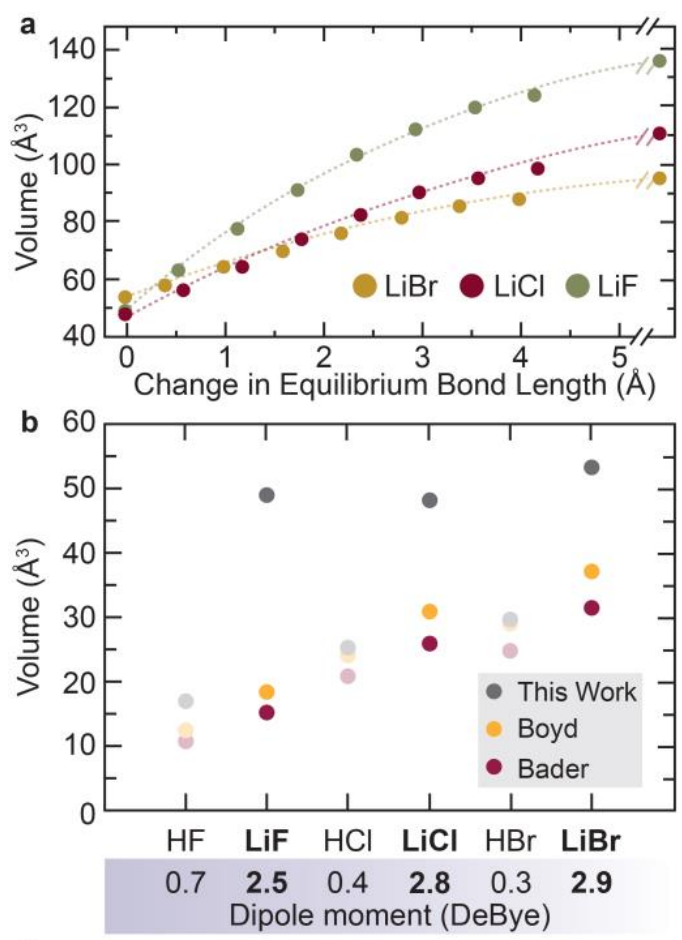

C

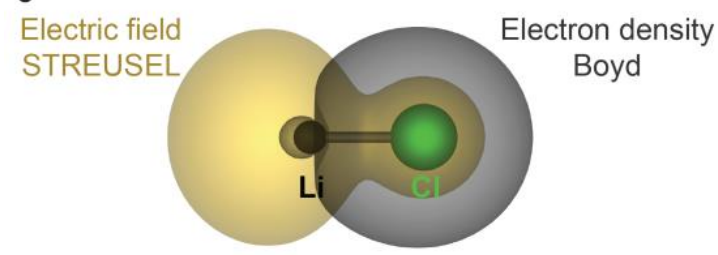

Figure 4 a) STREUSEL volumes for lithium halides at arbitrarily expanded bond lengths. At infinite separation the volumes are equivalent to $\mathrm{Li}^{+}$and the corresponding halide anion. Even at $4 \AA$, separation the ions are affected by one another, as evidenced by reduced field size, and atomic volume. b) Comparison of molecular volumes for a series of geometrically equilibrated diatomic molecules with varying dipole moments. Divergence between electron density-based size metrics and STREUSEL depends on the system polarity and atomic electronegativity. c) The difference in size between Boyd and STREUSEL for LiCl.

To study this further, we compute the ionic radii for each of the elements in the periodic table, Figure S5, and compare our computed size to yet another conventional volumetric approach, presented by Shannon and Prewitt.51,52 The Shannon-Prewitt method shows a clear dependence on both the oxidation state of the ion, but convolutes a direct comparison through the inclusion of a number of inner sphere ligands (with a general relationship between increasing number of inner sphere ligands and reducing ionic radii). The Shannon-Prewitt radii for the first-row transition metals are presented in Figure 3a. Given our approach computes size in the absence of ligands, we expected that STREUSEL radii should be larger than those from Shannon-Prewitt (as the ligands shield the electric field), and hence surmise that STREUSEL volumes should only depend on the ratio of nuclear to valence charge, Figure $\mathbf{3 b}$ and $\mathbf{c}$. From our method, the key observation is that radii are proportional to atomic charge (e.g. the extent of polarization), not necessarily identity of atom. This contrasts with the Shannon-Prewitt finding that both $\mathrm{Ti}^{2+}$ and $\mathrm{Zn}^{2+}$ are larger than $\mathrm{Fe}^{3+}$. Another conclusion from these data is that the oxidation states appear in radial bands. For example, $\mathrm{Fe}^{2+} / \mathrm{Fe}^{3+}$ and $\mathrm{Co}^{2+} / \mathrm{Co}^{3+}$ have similar differences in radii. of course, these values will dramatically reduce in the presence of external fields, highlighting the Shannon-Prewitttype dependence on local coordination.

Bonded atoms. Like the Shannon-Prewitt method, an electric field-derived approach should show a change in chemical size depending on proximity to other electric fields (e.g. those produced by ligands, atoms, molecules, surfaces of materials etc.). For example, the field produced by a free $\mathrm{Li}^{+}$ should be larger than $\mathrm{Li}^{+}$in proximity of $\mathrm{Cl}^{-}$, simply because they interact with one another. We can demonstrate that the size of ions depends on atomic proximity through progressive increase in bond lengths of some simple diatomics $(\mathrm{LiF}, \mathrm{LiCl}$, $\mathrm{LiBr}$ ), Figure 4a. We compute the bonded atoms using CCSDfull ${ }^{49} /$ aug-cc-pVTZ ${ }^{50}$ as implemented in Gaussian09.62 The basis was selected to both enable tractable calculations, with an ideal tradeoff between number of basis functions and accuracy (a comparison of basis sets is presented in Figure S2).

Here, the volumes of $\mathrm{LiF}, \mathrm{LiCl}$, and $\mathrm{LiBr}$ increase with increasing bond length, inevitably converging to the size of the sum of $\mathrm{Li}^{+}$and $\mathrm{X}^{-}$, computed either separately or very far apart within the same calculation $(>10 \AA$, to exceed the long-range cation interactions ${ }^{64,65}$ ). While the trend of increasing size with increasing interatomic separation is common to both the vdW and STREUSEL approaches, Figure S7, the volumetric trend of the dissociated lithium halides reveals a trend in opposition with the atomic size trends based on electron density. STREUSEL predicts that $\mathrm{F}^{-}>\mathrm{Cl}^{-}>\mathrm{Br}^{-}$, for the same reasons presented in Figure 3: the ratio of core charge to valence electrons is larger for the smaller, ionized atoms. Noting that this is at odds with the generally accepted metric that $\mathrm{Br}^{-}$is the largest of the three, this serves as a prime example of the unique perspective provided by the electric field; it is an inherent measure of affected size, and seemingly follows the general trend offered by Lewis' hard/soft acid/base theory (i.e. soft ions are indicative of small fields).

We can further highlight this relationship by examining the predicted volumes of $\mathrm{HF}, \mathrm{HCl}, \mathrm{HBr}$, in addition to the lithium halides, Figure $\mathbf{4 b}$. Here, there are two key observations; i) the acidic halides have near-zero dipole moment, resulting in volumes predicted by STREUSEL to align more closely with electron density-based methods (similar to data shown in Figure 2a for charge neutral atoms) and, ii) large dipole moments result in significant increases in volume, and a significant shift in the regions of the molecule producing this volume, Figure 4c. This comparison highlights the alternative affected volume metric offered by the electric fieldderived size, which is exacerbated for polarized bonded systems. Several other examples are presented in Figure S8.

DFT and applications to complex systems. While it should be clear that the electric field provides a conceptually different analysis of volume and radius in chemical systems, the utility of the presented approach is hitherto somewhat limited by the dependence on using CCSD-full/aug-cc-pVTZ. The following section hence seeks to identify alternate, less computationally intensive DFT-based methods that provide reliable size comparisons that can be used for large molecules and materials. Such an approach would boast the benefit of 
increased time-to-solution, and the ability to be applied to more diverse chemical systems.

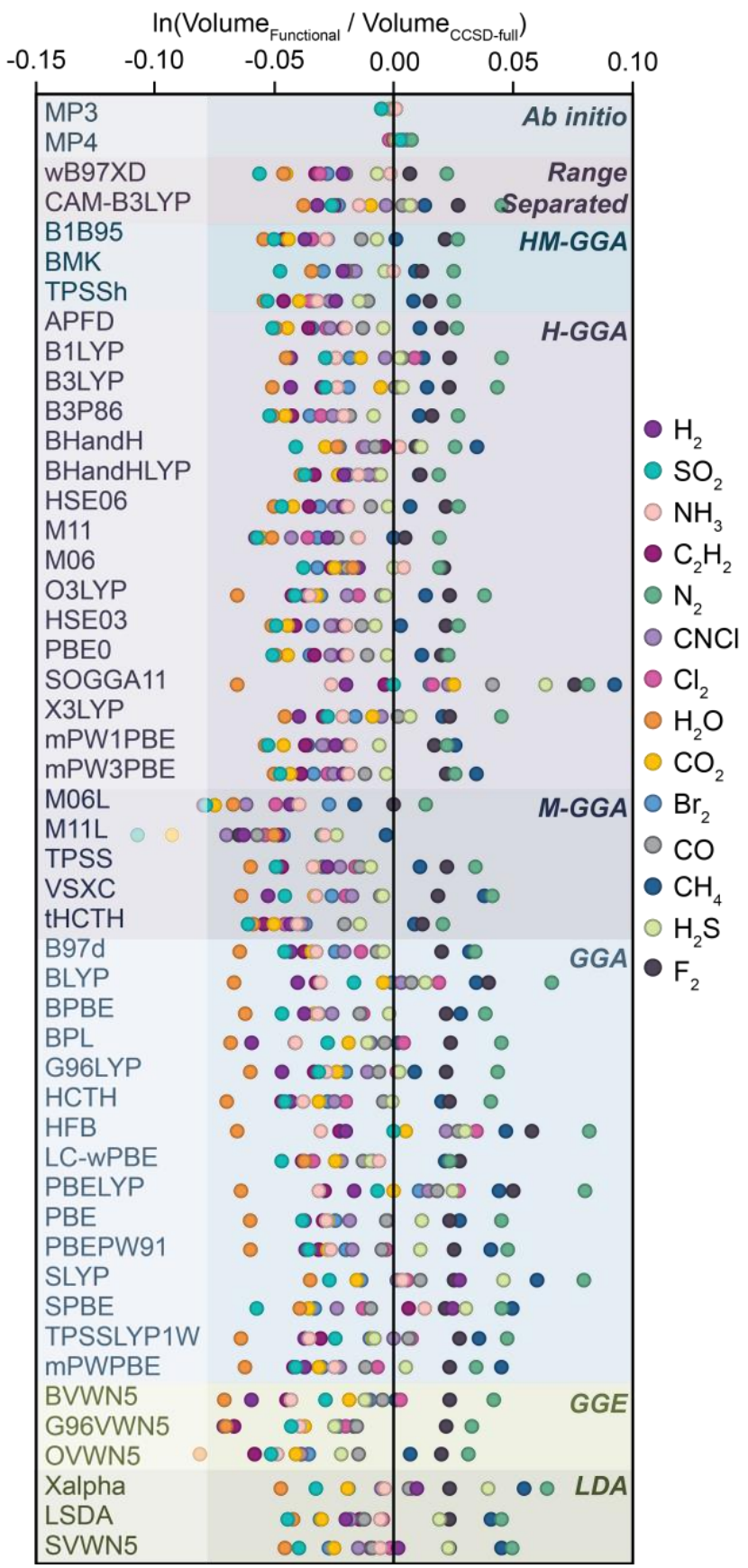

Figure 5 The calculated sizes for the small molecule systems shown, compared to the volumes computed using the structure and volume from CCSD-full. The $\mathrm{x}$-axis presents the natural logarithm of the volume fraction, y-axis groups functionals based on their electronic structure method.

As with all electronic structure methods, the size and shape of molecules depends on both the functional and basis set used for geometric equilibration. For this purpose, our basis remains constant (aug-cc-pVTZ), and the electronic structure method is explored. In a recent assessment of 128 DFT functionals, the authors canvassed their performance for recovery of total energy and, separately, electron density. ${ }^{49}$
That paper highlighted a significant energetic dependence on functional, and the disparity between energetic comparison and density comparison (which indirectly affects the shape of molecules). The latter is true because atomic position is determined by electron density, and electron density is determined by atomic position (i.e. the self-consistent field and geometry optimization routine), there is a dependence on DFT functional/ab initio method, and its impact on the size of the chemical system. ${ }^{66}$ In this context, we are interested in molecule volumes, surface areas, and shape, with energetics playing a secondary role (although there are certainly obvious future studies that may harness the energetics of interactions from electric field overlap).

To arrive at an ideal method to recover size using STREUSEL, we examined fifteen neutral molecules $\left(\mathrm{Ne}, \mathrm{H}_{2}, \mathrm{~N}_{2}\right.$, $\mathrm{F}_{2}, \mathrm{Cl}_{2}, \mathrm{Br}_{2}, \mathrm{H}_{2} \mathrm{O}, \mathrm{H}_{2} \mathrm{~S}, \mathrm{NH}_{3}, \mathrm{CO}, \mathrm{CO}_{2}, \mathrm{CH}_{4}, \mathrm{C}_{2} \mathrm{H}_{2}, \mathrm{CNCl}$, and $\mathrm{SO}_{2}$ ) using forty-nine DFT and $a b$ initio methods. The selection of small molecules spans a range of polarizations and oxidation states. While the methods in Figure 5 only canvas the upper rungs of Jacob's ladder ${ }^{67,68}$, eight electronic structure classes are included ( $a b$ initio, generalized gradient approximation (GGA), generalized gradient exchange (GGE), hybrid-GGA (HGGA), hybrid-meta-GGA (HM-GGA), local-density approximation (LDA), meta-GGA (M-GGA), and range separated functionals). Like before, CCSD-full ${ }^{58-61}$ is used as our geometric and electronic reference for the exact solution, and each molecule was geometrically equilibrated using the stated functional. Its volume, radius, surface area, and other topological properties were then computed from the electrostatic potential. 
As we ascend Jacob's Ladder, higher level DFT functionals do not immediately appear to outperform lower-level ones, excluding $a b$ initio methods, which are highly accurate. Generally, GGE, HM-GGA, and M-GGA functionals appear to systematically underestimate molecular volumes (revealed in the mean volume deviations, Figure S9), and $F_{2}$ and $\mathrm{Ne}$ are anomalously overestimated independent of DFT method. It should be noted that the axis in Figure $\mathbf{5}$ is deliberately presented to show differences between functionals, and perhaps a better comparison is achieved by examining average mean deviations between functional classes, Figure S9. There, it becomes apparent that there is no clear preferred functional, at least not predictably so. It also highlights one plausible reason why the vdW approach has been widely adopted there is very little dependence on minor fluctuations in bond length in predicted molecule volume (see Table S2 with reference to Figure S9). Also, the mean absolute error for molecules optimized with B3LYP, a widely used functional for small organic molecules, appears to reliably approximate that of CCSD-full. However, the electric field does provide additional and complementary insights and hence with no clear victor. We recommend a pragmatic approach to functional selection: a balance of accuracy and time-tosolution. With respect to Figure $\mathbf{5}$ and $\mathbf{S 9}$, we are guided to favor functionals and $a b$ initio methods displaying lowest mean absolute deviation within their functional class: MP3 and MP4 (ab initio); CAM-B3LYP (range separated); BMK (HMGGA): BHandH, BHandHLYP, X3LYP, B1LYP, and B3LYP (HGGA); TPSS and VSXC (M-GGA), BPL, G96LYP, and TPSSLYP1W (GGA); BVWN5 (GGE); Xalpha (LDA). With a tractable functional at hand, we can now tackle more sophisticated examples of the utility of electric field-based molecular topologies; molecular ions in ionic liquids, and void spaces in solids.

In the case of ionic liquids, molar volume is a critical parameter which is thought to govern the physical properties of the bulk, including density, viscosity, and so forth. ${ }^{69}$ The density and molar volume should depend on the size of the ions, which itself may depend on whether they are computed together or separately. It may be useful to predict the packing volumes from small molecule calculations, ideally of the free ions themselves, to overcome sampling of the various geometric configurations. By way of example, we consider two simple chemical systems, $[\mathrm{BMIM}]\left[\mathrm{VCl}_{4}\right]$ and $[\mathrm{BMIM}]_{2}\left[\mathrm{CoCl}_{4}\right]$ (BMIM = 1-butyl-3-methylimidazolium, Table 2). ${ }^{70}$ Using the geometries computed using M06L (one of the better performing M-GGA methods), several volume metrics are presented. Both STREUSEL and vdW appear to predict similar sizes of the free ions, while electron density methods significantly under-estimate the sizes of the molecular ions.

From Figure 4, one may initially assume that the free molecular ions would always be larger than their paired analogues. Yet, within polynuclear systems there are three competing phenomena determining the size of the ions;

i) anionic and cationic electric fields interfering with one another, leading to a decrease in net volume,

ii) a change in molecular shape due to intermolecular interactions, leading to an unpredictable change in shape,

iii) the possibility of the external field polarizing the neighboring molecule, leading to an increase in volume.

Thus, to explore these nuances, we further computed the same ionic liquid components as ion pairs in an equilibrium geometry, entry 2 of Table 2 . There, the vdW, Boyd, and Bader methods show a dramatic decrease in volume, due to intermolecular overlap between the species. However, STREUSEL appears to recover similar volumes for the molecules computed separately or together $(<4 \%$ change $)$. Unlike those data presented in Figure 4, the lack of change is likely because the atoms carrying the formal increased valence are heavily buried within the molecule, thereby being shielded from external fields. Perhaps this finding may prove to be a useful design principle for assessing volumes, densities, and other 3D chemical properties a priori, and feed into computer accelerated materials discovery ${ }^{71-73}$ by rapidly screening molecular libraries for ideal multimolecular aggregates, forgoing arduous sampling of the multitude of intermolecular interacting geometries.

\section{CONCLUSION AND OUTLOOK}

The calculation of atomic, molecular, and material size has always been defined by an arbitrary cutoff, where one chemical system ends, and another begins. In prior approaches, the determination of atomic radii, volume, and associated surface area, has depended on electron density (either experimental or from quantum chemical simulation).

Table 2. Boyd, Bader, Batsanov and STREUSEL-derived molecular volumes $\left(\AA^{3}\right)$ for the $[B M I M]_{2}\left[\mathrm{CoCl}_{4}\right]$ ionic liquid, BMIM $\equiv$ 1-butyl-3-methylimidazolium. The sum of single molecule volumes for the individual constituents $\left([\mathrm{BMIM}]^{+},\left[\mathrm{CoCl}_{4}\right]^{2-}\right)$ are presented, as well an equilibrium ion pair. The percent difference (\%) is presented for each ionic liquid model.

\begin{tabular}{|c|c|c|c|c|c|c|}
\hline & & Boyd & Bader & STREUSEL & Batsanov & Dipole \\
\hline \multirow[t]{3}{*}{ Single Molecules } & [BMIM] $^{+}$ & \multirow{2}{*}{$\begin{array}{l}73.98 \\
64.37\end{array}$} & \multirow{2}{*}{$\begin{array}{l}66.62 \\
58.24\end{array}$} & \multirow{2}{*}{$\begin{array}{l}158.8 \\
87.94\end{array}$} & \multirow{2}{*}{$\begin{array}{l}161.3 \\
107.3\end{array}$} & 5.32 \\
\hline & {$\left[\mathrm{VCl}_{4}\right]^{-}$} & & & & & 0.055 \\
\hline & {$[\mathrm{BMIM}]^{+}+\left[\mathrm{VCl}_{4}\right]^{-}$} & \multirow{2}{*}{$\begin{array}{l}138.3 \\
114.8 \\
\end{array}$} & 124.9 & \multirow{2}{*}{$\begin{array}{l}246.7 \\
247.1 \\
\end{array}$} & \multirow{2}{*}{$\begin{array}{ll}268.6 & \mathbf{2 1 \%} \\
217.6 & \end{array}$} & \\
\hline Ion pair & {$[\mathrm{BMIM}]\left[\mathrm{VCl}_{4}\right]$} & & 105.1 & & & \\
\hline \multirow[t]{3}{*}{ Single Molecules } & [BMIM] $^{+}$ & \multirow{2}{*}{$\begin{array}{l}73.98 \\
68.35\end{array}$} & \multirow{2}{*}{$\begin{array}{l}66.62 \\
61.95\end{array}$} & \multirow{2}{*}{$\begin{array}{l}158.8 \\
86.63\end{array}$} & \multirow{2}{*}{$\begin{array}{l}161.3 \\
107.6\end{array}$} & 5.32 \\
\hline & {$\left[\mathrm{CoCl}_{4}\right]^{2-}$} & & & & & 0.403 \\
\hline & $2\left[\mathrm{BMIM}^{+}+\left[\mathrm{CoCl}_{4}\right]^{2-}\right.$ & \multirow{2}{*}{$\begin{array}{ll}216.3 \\
173.1\end{array}$} & \multirow{2}{*}{$\begin{array}{ll}195.2 & \mathbf{2 0 \%} \\
159 . \epsilon & \end{array}$} & \multirow{2}{*}{$\begin{array}{l}404.2 \\
390.1\end{array}$} & \multirow{2}{*}{$\begin{array}{ll}430.2 & \mathbf{3 8 \%} \\
293.6 & \end{array}$} & \\
\hline Ion pair & {$[\mathrm{BMIM}]_{2}\left[\mathrm{CoCl}_{4}\right]$} & & & & & \\
\hline
\end{tabular}


Yet the limitations of these models have been highlighted by comparison of highly polarized systems, with the most striking being a difference in affected volume of free cations. Indeed, the contrast between the work presented herein, and the van der Waals metric is the description of the atomic surface being defined by a field smaller than the thermal energy provided at near absolute $0 \mathrm{~K}$. The value of the latter multifold: it allows for an assessment of how large an area is affected by the presence of the ion (an effect that may prove to be significant in applied electrochemistry ${ }^{74}$, and other complex systems ${ }^{75,76}$ ), it allows for direct estimates of dispersion interactions (by redefining the cutoff to account for temperature effects such that materials can interact via their fields more strongly than $k_{\mathrm{B}} \mathrm{T}$ ), and by considering the contribution to surface area and volume may indeed be defined by the region created by the cation. And while our method undoubtedly does not replace the vdW radius or other size definitions, it does provide an alternative framework to contemplate the boundaries of chemistry.

\section{ASSOCIATED CONTENT}

\section{Supporting Information}

The Supporting Information is available free of charge on the ACS Publications website.

Volumetric pixels convergence tests, extended small molecule functional screening, and basis set convergence.

\section{AUTHOR INFORMATION}

\section{Corresponding Author}

Christopher H. Hendon - Department of Chemistry and Biochemistry, University of Oregon, Eugene, OR, 97403; orcid.org/0000-0002-7132-768X; Email: chendon@uoregon.edu

\section{Authors}

Austin M. Mroz - Department of Chemistry and Biochemistry, University of Oregon, Eugene, OR, 97403; orcid.org/0000-0003-4166-8093

Joshua Davis - Department of Chemistry and Biochemistry, University of Oregon, Eugene, OR, 97403

\section{Author Contributions} authors.

The manuscript was written through contributions of all

\section{Funding Sources}

NSF Division of Materials Research under grant no. DMR1956403.

\section{ACKNOWLEDGMENT}

This work is based upon work supported by the National Science Foundation through the Division of Materials Research under grant no. DMR-1956403. CHH also acknowledges the Research Corporation for Science Advancement (Cottrell Award). We are grateful for the continued access to the Extreme Science and Engineering Discovery Environment (XSEDE), which is supported by the
National Science Foundation [ACI-1548562] and the PICS Coeus High Performance Computer, which is supported by the National Science Foundation [1624776]. The authors would also like to thank Prof. Roald Hoffmann, Prof. Martin Rahm, and Prof. Carl K. Brozek for their thoughtful comments on the manuscript, as well as Dr. Andrew Tarzia for their valuable suggestions and discussion. We further appreciate the PLATON simulations performed by Kevin Fabrizio.

\section{REFERENCES}

(1) Yue, X.; Taraban, M. B.; Hyland, L. L.; Yu, Y. B. Avoiding Steric Congestion in Dendrimer Growth through Proportionate Branching: A Twist on Da Vinci's Rule of Tree Branching. J. Org. Chem. 2012, 77, 8879-8887.

(2) Luo, P.; Dinnocenzo, J. P.; Merkel, P. B.; Young, R. H.; Farid, S. Bimolecular Electron Transfers That Deviate from the Sandros-Boltzmann Dependence on Free Energy: Steric Effect. J. Org. Chem. 2012, 77, 1632-1639.

(3) Durand, D. J.; Fey, N. Computational Ligand Descriptors for Catalyst Design. Chem. Rev. 2019, 119, 6561-6594.

(4) Mohamed, R. K.; Peterson, P. W.; Alabugin, I. V. Concerted Reactions That Produce Diradicals and Zwitterions: Electronic, Steric, Conformational, and Kinetic Control of Cycloaromatization Processes. Chem. Rev. 2013, 113, 7089-7129.

(5) Ravelli, D.; Fagnoni, M.; Fukuyama, T.; Nishikawa, T.; Ryu, I. Site-Selective C-H Functionalization by Decatungstate Anion Photocatalysis: Synergistic Control by Polar and Steric Effects Expands the Reaction Scope. ACS Catal. 2018, 8, 701-713.

(6) Wagner, J. P.; Schreiner, P. R. London Dispersion in Molecular Chemistry: Reconsidering Steric Effects. Angew. Chem. Int. Ed. 2015, 54, 12274-12296.

(7) Poli, G.; Martinelli, A.; Tuccinardi, T. Reliability Analysis and Optimization of the Consensus Docking Approach for the Development of Virtual Screening Studies. J. Enzyme Inhib. Med. Chem. 2016, 31, 167-173.

(8) Ue, M.; Murakami, A.; Nakamura, S. A Convenient Method to Estimate Ion Size for Electrolyte Materials Design. J. Electrochem. Soc. 2002, 149, A1385-A1388.

(9) Rahm, M.; Hoffmann, R.; Ashcroft, N. Atomic and Ionic Radii of Elements 1-96. Chem. Eur. J. 2016, 22, 14625-14632.

(10) Brunauer, S.; Emmett, P. H.; Teller, E. Adsorption of Gases in Multimolecular Layers. J. Am. Chem. Soc. 1938, 60, 309319.

(11) Thomsen, R.; Christensen, M. H. MolDock: A New Technique for High-Accuracy Molecular Docking. J. Med. Chem. 2006, 49, 3315-3321.

(12) Meng, X.-Y.; Zhang, H.-X.; Mezei, M.; Cui, M. Molecular Docking: A Powerful Approach for Structure-Based Drug Discovery. Curr. Comp. Aid. Drug Des. 2011, 7, 146-157.

(13) Meyer, L. Die Natur Der Chemischen Elemente Als Function Ihrer Atomgewichte. Ann. Chem. Pharm. 1870, 354-364.

(14) Bragg, W. L. The Arrangement of Atoms in Crystals. Philos. Mag. 1920, 40, 169-189.

(15) Pauling, L. C. The Nature of the Chemical Bond and the Structure of Moleculesa Nd Crystals. In An Introduction to Modern Structural Chemistry; Cornell University Press: Ithaca, NY, 1960; p 644.

(16) Bondi, A. Van Der Waals Volumes and Radii. J. Phys. Chem. 1964, 68, 441-451.

(17) Bondi, A. Van Der Waals Volumes and Radii of Metals in Covalent Compounds. J. Phys. Chem. 1966, 70, 3006-2007.

(18) Batsanov, S. Van Der Waals Radii of Elements. Inorg. Mater. 2001, 37, 1031-1046.

(19) Cordero, B.; Gomez, V.; Platero-Prats, A. E.; Reves, M.; Echeverria, J.; Cremades, E.; Barragan, F.; Alvarez, S. Covalent Radii Revisited. Dalton Trans. 2008, 2832-2838. 
(20) Alvarez, S. A Cartography of the van Der Waals Territories. Dalton Trans. 2013, 42, 8627-8636.

(21) Ghosh, D. C.; Biswas, R. Theoretical Calculation of Absolute Radii of Atoms and Ions. Part. 1 The Atomic Radii. Int. J. Mol. Sci. 2002, 3, 87-113.

(22) Delgado-Andrade, C.; Morales, F. J. Unraveling the Contribution of Melanoidins to the Antioxidant Activity of Coffee Brews. J. Agric. Food Chem. 2005, 53, 1403-1407.

(23) Vincze, J.; Valiskó, M.; Boda, D. The Nonmonotonic Concentration Dependence of the Mean Activity Coefficient of Electrolytes Is a Result of a Balance between Solvation and IonIon Correlations. J. Chem. Phys. 2010, 133, 154507.

(24) Fraenkel, D. Computing Excess Functions of Ionic Solutions: The Smaller-Ion Shell Model versus the Primitive Model. 1. Activity Coefficients. J. Chem. Theory Comput. 2015, 11, 178-192.

(25) Schreiner, P. R.; Chernish, L. V.; Gunchenko, P. A.; Tikhonchuk, E. Yu.; Hausmann, H.; Serafin, M.; Schlecht, S.; Dahl, J. E. P.; Carlson, R. M. K.; Fokin, A. A. Overcoming Lability of Extremely Long Alkane Carbon-Carbon Bonds through Dispersion Forces. Nature 2011, 477, 308-311.

(26) Ishigaki, Y.; Shimajiri, T.; Takeda, T.; Katoono, R.; Suzuki, T. Longest C-C Single Bond among Neutral Hydrocarbons with a Bond Length beyond $1.8 \AA$. Chem 2018, 4, 795-806.

(27) Li, J.; Pang, R.; Li, Z.; Lai, G.; Xiao, X.-Q.; Muller, T. Exceptionally Long C-C Single Bonds in Diamino-o-Carborane as Induced by Negative Hyperconjugation. Angew. Chem. Int. Ed. 2019, 58, 1397-1401.

(28) Bak, K. L.; Gauss, J.; Jørgensen, P.; Olsen, J.; Helgaker, T.; Stanton, J. F. The Accurate Determination of Molecular Equilibrium Structures. J. Chem. Phys. 2001, 114, 6548-6556.

(29) Clementi, E.; Raimondi, D. L. Atomic Screening Constants from SCF Functions. J. Chem. Phys. 1963, 38, 2686.

(30) Clementi, E.; Raimondi, D. L.; Reinhardt, W. P. Atomic Screening Constants from SCF Functions. II. Atoms with 37-86 Electrons. J. Chem. Phys. 1967, 47, 1300-1307.

(31) Slater, J. C. Atomic Shielding Constants. Phys. Rev. 1930, $36,57$.

(32) Zunger, A.; Cohen, M. L. First-Principles NonlocalPseudopotential Approach in the Density-Functional Formalism: Development and Application to Atoms. Phys. Rev. B 1978, 18, 5449-5472.

(33) Zunger, A.; Cohen, M. L. First-Principles NonlocalPseudopotential Approach in the Density-Functional Formalism. II. Application to Electronic and Structural Properties of Solids. Phys. Rev. B 1979, 20, 4082-4108.

(34) Zhang, S. B.; Cohen, M. L.; Phillips, J. C. Relativistic Screened Orbital Radii. Phys. Rev. B 1987, 36, 5861-5867.

(35) Nath, S.; Chattaqcharya, S.; Chattaraj, P. K. Density Functional Calculation of a Characteristic Atomic Radius. Theochem 1995, 331, 267-279.

(36) Putz, M. V.; Russo, N.; Sicilia, E. Atomic Radii Scale and Related Size Properties from Density Functional Electronegativity Formulation. J. Phys. Chem. A 2003, 107, 54615465 .

(37) Hollett, J. W.; Kelly, A.; Poirier, R. A. Quantum Mechanical Size and Steric Hindrance. J. Phys. Chem. A 2006, 110, 13884-13888.

(38) Bader, R. F. W.; Henneker, W. H.; Cade, P. E. Molecular Charge Distributions and Chemical Binding. J. Chem. Phys. 1967, 46, 3341-3363.

(39) Boyd, R. The Relative Sizes of Atoms. J. Phys. B: Atom. Molec. Phys. 1977, 10, 2283.

(40) Kohn, W.; Sham, L. J. Self-Consistent Equations Including Exchange and Correlation Effects. Phys. Rev. 1965, 140, A1133-A1138.

(41) Kohn, W.; Becke, A. D.; Parr, R. G. Density Functional Theory of Electronic Structure. J. Phys. Chem. 1996, 100, 1297412980.
(42) Lee, C.; Yang, W.; Parr, R. G. Development of the ColleSalvetti Correlation-Energy Formula into a Functional of the Electron Density. Phys. Rev. B 1988, 37, 785-789.

(43) Perdew, J.; Burke, K.; Ernzerhof, M. Generalized Gradient Approximation Made Simple. Phys. Rev. Lett. 1996, 77, 3865-3868.

(44) Perdew, J.; Burke, K.; Ernzerhof, M. Errata: Generalized Gradient Approximation Made Simple. Phys. Rev. Lett. 1997, 78, 1396.

(45) Politzer, P.; Murray, J. S. The Fundamental Nature and Role of the Electrostatic Potential in Atoms and Molecules. Theor. Chem. Acc. Theory Comput. Model. Theor. Chim. Acta 2002, 108, 134-142.

(46) https://github.com/hmsoregon/STREUSEL (accessed 09/18/2021)

(47) Kannemann, F. O.; Becke, A. D. Van Der Waals Interactions in Density-Functional Theory: Intermolecular Complexes. J. Chem. Theory Comput. 2010, 6, 1081-1088.

(48) Different Types of Interactions in Macromolecules. In Compendium of Biophysics; John Wiley \& Sons, Inc.: Hoboken, NJ, USA, 2017; pp 109-116.

(49) Medvedev, M. G.; Bushmarinov, I. S.; Sun, J.; Perdew, J. P.; Lyssenko, K. A. Density Functional Theory Is Straying from the Path toward the Exact Functional. Science 2017, 355, 49-52.

(50) Dunning, T. H. Gaussian Basis Sets for Use in Correlated Molecular Calculations. I. The Atoms Boron through Neon and Hydrogen. J. Chem. Phys. 1989, 90, 1007-1023.

(51) Shannon, R. D. Revised Effective Ionic Radii and Systematic Studies of Interatomic Distances in Halides and Chalcogenides. Acta Crystallogr. Sect. A 1976, 32, 751-767.

(52) Shannon, R. D.; Prewitt, C. T. Effective Ionic Radii in Oxides and Fluorides. Acta Crystallogr. B 1969, 25, 925-946.

(53) Ouyang, R. Exploiting Ionic Radii for Rational Design of Halide Perovskites. Chem. Mater. 2020, 32, 595-604.

(54) Agmon, N. Isoelectronic Theory for Cationic Radii. J. Am Chem. Soc. 2017, 139, 15068-15073.

(55) Slater, J. C. Atomic Radii in Crystals. J. Chem. Phys. 1964, 41, 3199-3204.

(56) Batsanov, S. S. Determination of Ionic Radii from Metal Compressibilities. J. Struct. Chem. 2004, 45, 896-899.

(57) Pyykko, P. Additive Covalent Radii for Single-, Doubleand Triple-Bonded Moleculars and Tetrahedrally Bonded Crystals: A Summary. J. Phys. Chem. A 2015, 119, 2326-2337.

(58) Scuseria, G. E.; Schaefer, H. F. Is Coupled Cluster Singles and Doubles (CCSD) More Computationally Intensive than Quadratic Configuration Interaction (QCISD)? J. Chem. Phys. 1989, 90, 3700-3703.

(59) Scuseria, G. E.; Janssen, C. L.; Schaefer, H. F. An Efficient Reformulation of the Closed-shell Coupled Cluster Single and Double Excitation (CCSD) Equations. J. Chem. Phys. 1988, 89, 7382-7387.

(60) Purvis, G. D.; Bartlett, R. J. A Full Coupled-cluster Singles and Doubles Model: The Inclusion of Disconnected Triples. Chem. Phys. 1982, 76, 1910-1918.

(61) Advances in Chemical Physics: LeFebvre/Advances; LeFebvre, R., Moser, C., Eds.; Advances in Chemical Physics; John Wiley \& Sons, Inc.: Hoboken, NJ, USA, 1969.

(62) Frisch, M.; Schlegel, H.; Scuseria, G.; Robb, M.; Cheeseman, J.; Scalmani, G.; Barone, V.; Petersson, G.; Nakatsuji, H.; Li, X. et al. Gaussian09; Gaussian Inc. Wallingford CT, 2009.

(63) Kafader, J. O.; Ray, M.; Jarrold, C. C. Low-Lying Electronic Structure of EuH, EuOH, and EuO Neutrals and Anions Determined by Anion Photoelectron Spectroscopy and DFT Calculations. J. Chem. Phys. 2015, 143, 034305.

(64) Park, K. C.; Tsukahara, T. Quantitative Evaluation of Long-Range and Cooperative Ion Effect on Water in Polyamide Network. J. Phys. Chem. B 2019, 123, 2948-2955.

(65) Gebbie, M. A.; Smith, A. M.; Dobbs, H. A.; Lee, A. A.; Warr, G. G.; Banquy, X.; Valtiner, M.; Rutland, M. W.; Israelachvili, J. N.; 
Perkin, S.; Atkin, R. Long Range Electrostatic Forces in Ionic Liquids. Chem. Commun. 2017, 53, 1214-1224.

(66) McAnanama-Brereton, S.; Waller, M. P. Rational Density Functional Selection Using Game Theory. J. Chem. Inf. Model. 2018, 58, 61-67.

(67) Mattsson, A. E. In Pursuit of the "Divine" Functional. Science 2002, 298, 759-760.

(68) Mancuso, J. L.; Mroz, A. M.; Le, K. N.; Hendon, C. H. Electronic Structure Modeling of Metal-Organic Frameworks. Chem. Rev. 2020, 120, 8641-8715.

(69) Chaban, V. V.; Andreeva, N. A.; Fileti, E. E. Graphene/Ionic Liquid Ultracapacitors: Does Ionic Size Correlate with Energy Storage Performance? New J. Chem. 2018, 42, 18409-18417.

(70) LeRoy, M. A.; Mroz, A. M.; Mancuso, J. L.; Miller, A.; Van Cleve, A.; Check, C.; Heinz, H.; Hendon, C. H.; Brozek, C. K. PostSynthetic Modification of Ionic Liquids Using Ligand-Exchange and Redox Coordination Chemistry. J. Mater. Chem. A 2020, 8, 22674-22685.

(71) Chandrasekaran, A.; Kamal, D.; Batra, R.; Kim, C.; Chen, L.; Ramprasad, R. Solving the Electronic Structure Problem with Machine Learning. Npj Comput. Mater. 2019, 5, 22.
(72) Grisafi, A.; Fabrizio, A.; Meyer, B.; Wilkins, D. M.; Corminboeuf, C.; Ceriotti, M. Transferable Machine-Learning Model of the Electron Density. ACS Cent. Sci. 2019, 5, 57-64.

(73) Tsubaki, M.; Mizoguchi, T. Quantum Deep Field: DataDriven Wave Function, Electron Density Generation, and Atomization Energy Prediction and Extrapolation with Machine Learning. Phys. Rev. Lett. 2020, 125, 206401.

(74) Liu, M.; Pang, Y.; Zhang, B.; De Luna, P.; Voznyy, O.; Xu, J.; Zheng, X.; Dinh, C. T.; Fan, F.; Cao, C.; de Arquer, F. P. G.; Safaei, T. S.; Mepham, A.; Klinkova, A.; Kumacheva, E.; Filleter, T.; Sinton, D.; Kelley, S. O.; Sargent, E. H. Enhanced Electrocatalytic $\mathrm{CO}_{2}$ Reduction via Field-Induced Reagent Concentration. Nature 2016, 537, 382-386.

(75) Sutton, E. C.; McDevitt, C. E.; Prochnau, J. Y.; Yglesias, M. V.; Mroz, A. M.; Yang, M. C.; Cunningham, R. M.; Hendon, C. H.; DeRose, V. J. Nucleolar Stress Induction by Oxaliplatin and Derivatives. J. Am. Chem. Soc. 2019, 141, 18411-18415.

(76) McDevitt, C. E.; Yglesias, M. V.; Mroz, A. M.; Sutton, E. C.; Yang, M. C.; Hendon, C. H.; DeRose, V. J. Monofunctional Platinum(II) Compounds and Nucleolar Stress: Is Phenanthriplatin Unique? JBIC J. Biol. Inorg. Chem. 2019, 24, 899908

\section{Graphical Abstract}

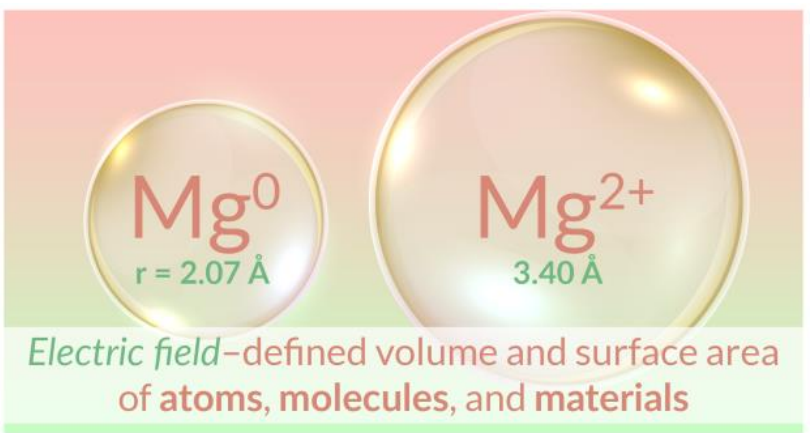

